\title{
Sturdy frames of type $(2,2)$ algebras and their applications to semirings
}

\author{
by \\ X. Z. Zhao (Xi'an), Y. Q. Guo (Beibei) and \\ K. P. Shum (Hong Kong)
}

\begin{abstract}
We introduce sturdy frames of type $(2,2)$ algebras, which are a common generalization of sturdy semilattices of semigroups and of distributive lattices of rings in the theory of semirings. By using sturdy frames, we are able to characterize some semirings. In particular, some results on semirings recently obtained by Bandelt, Petrich and Ghosh can be extended and generalized.
\end{abstract}

1. Introduction. A strong semilattice of semigroups $S_{\alpha}$, denoted by $\left[Y ; S_{\alpha} ; \psi_{\alpha, \beta}\right]$, is called a sturdy semilattice of semigroups if the set of structure homomorphisms $\psi_{\alpha, \beta}$ which map $S_{\alpha}$ into $S_{\beta}$ for $\alpha \geq \beta$ is a transitive system and in addition, all mappings $\psi_{\alpha, \beta}$ are one-to-one. We usually denote a sturdy semilattice of semigroups $S_{\alpha}$ by $\left\langle Y ; S_{\alpha} ; \psi_{\alpha, \beta}\right\rangle$. Sturdy semilattices of semigroups were first introduced by Petrich and Reilly [7]. They are an important tool for the construction of some semigroups of certain varieties. In the investigation of semirings, S. Ghosh [2] has recently introduced distributive lattices of rings. On the other hand, in the study of the structure of idempotent semirings, Sen, Guo and Shum [9] have defined the concept of a distributive lattice of semirings.

In this paper, we introduce the concept of a sturdy frame of type $(2,2)$ algebras which is a common generalization of the above two concepts. We will show that by using sturdy frames of type $(2,2)$ algebras, we can characterize some important varieties of semirings. In particular, some results on semirings obtained by H. J. Bandelt and M. Petrich [1] and S. Ghosh [2] are extended and generalized.

2000 Mathematics Subject Classification: 16Y60, 08B25, $20 \mathrm{M} 07$.

Key words and phrases: type $(2,2)$ algebras, semirings, frames, Mal'tsev product.

The research of Y. Q. Guo is supported by a grant of NSF, China \# 10071068 and a grant of Yunnan Provincial Applied Fundamental Research Foundation \# 96a001z.

The research of K. P. Shum is partially supported by a UGC(HK) grant \#2160176(2002/2003). 
By a frame $B$, we mean a type $(2,2)$ algebra $(B,+, \cdot)$ endowed with an upper semilattice order $\leq$ satisfying the following frame conditions:

$$
a+b \leq a \vee b, \quad a b \leq a \vee b,
$$

for all $a, b \in B$, where $a \vee b=\operatorname{lub}(a, b)$.

Clearly, a lattice is a frame. Also any semilattice $Y$ can be regarded as a frame because we can consider it as a type $(2,2)$ algebra on which addition and multiplication coincide and the partial order is an upper semilattice order $\leq$ defined by

$$
a \leq b \Leftrightarrow a b=b
$$

for any $a, b \in Y$.

By an upper semilattice type $(2,2)$ algebra, we mean a semilattice ordered type $(2,2)$ algebra $(B,+, \cdot)$ satisfying the identity

$$
x+x \approx x \cdot x \approx x
$$

with an upper semilattice order $\leq$ compatible with both addition and multiplication on $B$. Let $(B,+, \cdot, \leq)$ be an upper semilattice type $(2,2)$ algebra. Then $B$ is clearly an upper semilattice. Since $a \leq a \vee b$ for any $a, b \in B$, we have $a+b \leq a+a \vee b \leq a \vee b+a \vee b=a \vee b$. Similarly, $a b \leq a \vee b$. Thus, $(B,+, \cdot, \leq)$ satisfies the frame conditions. In other words, an upper semilattice type $(2,2)$ algebra is always a frame.

The converse is not necessarily true; for example, let $S$ be a frame with the following additive reduct $(S,+)$ and multiplicative reduct $(S, \cdot)$ :

\begin{tabular}{c|ccc}
+ & $a$ & $b$ & 0 \\
\hline$a$ & $a$ & $b$ & $a$ \\
$b$ & $b$ & $b$ & $b$ \\
0 & $a$ & $b$ & 0
\end{tabular}

\begin{tabular}{c|ccc}
$\cdot$ & $a$ & $b$ & 0 \\
\hline$a$ & $a$ & $a$ & 0 \\
$b$ & $b$ & $b$ & 0 \\
0 & 0 & 0 & 0
\end{tabular}

together with an upper semilattice order defined by $u \leq v$ if and only if $u v=v u=u$. Then $S$ is not an upper semilattice type $(2,2)$ algebra because $a \vee b=0, b \leq 0$, but $b+a=b \not \leq a=0+a$.

Similar to the construction of a sturdy semilattice of semigroups, we can also define a sturdy frame of type $(2,2)$ algebras.

Let $\left\{\left(S_{\alpha},+, \cdot\right) \mid \alpha \in B\right\}$ be a family of disjoint type $(2,2)$ algebras indexed by a frame $B$. Let $\varphi_{\alpha, \beta}: S_{\alpha} \rightarrow S_{\beta}(\alpha \leq \beta)$ be a family of monomorphisms satisfying the following conditions: for any $\alpha, \beta, \gamma \in B$,

(i) $\varphi_{\alpha, \alpha}=1_{S_{\alpha}}$;

(ii) if $\alpha \leq \beta \leq \gamma$, then $\varphi_{\alpha, \beta} \varphi_{\beta, \gamma}=\varphi_{\alpha, \gamma}$;

(iii) if $\alpha \vee \beta \leq \gamma$, then

$$
\begin{aligned}
& S_{\alpha} \varphi_{\alpha, \gamma}+S_{\beta} \varphi_{\beta, \gamma} \subseteq S_{\alpha+\beta} \varphi_{\alpha+\beta, \gamma}, \\
& S_{\alpha} \varphi_{\alpha, \gamma} \cdot S_{\beta} \varphi_{\beta, \gamma} \subseteq S_{\alpha \beta} \varphi_{\alpha \beta, \gamma} .
\end{aligned}
$$


Now, we form the set union $S=\bigcup_{\alpha \in B} S_{\alpha}$ and define addition and multiplication on $S$ by

$$
\begin{aligned}
a+b & =\left(a \varphi_{\alpha, \alpha \vee \beta}+b \varphi_{\beta, \alpha \vee \beta}\right) \varphi_{\alpha+\beta, \alpha \vee \beta}^{-1}, \\
a b & =\left(a \varphi_{\alpha, \alpha \vee \beta} b \varphi_{\beta, \alpha \vee \beta}\right) \varphi_{\alpha \beta, \alpha \vee \beta}^{-1},
\end{aligned}
$$

for any $a \in S_{\alpha}, b \in S_{\beta}$. We can easily check that $(S,+, \cdot)$ forms a type $(2,2)$ algebra, denoted by $S=\left\langle B, \leq S_{\alpha}, \varphi_{\alpha, \beta}\right\rangle$. We call it the sturdy frame of the type $(2,2)$ algebras $S_{\alpha}$ on the frame $B$.

It is clear that sturdy semilattices of semigroups studied by Petrich and Reilly [7], distributive lattices of rings studied by S. Ghosh [2], and distributive lattices of semirings studied by other authors in [1], [4], [6], [9] are all special cases of sturdy frames of type $(2,2)$ algebras because semilattices and distributive lattices are frames and a semigroup can always be regarded as a type $(2,2)$ algebra on which addition and multiplication coincide. We will see that sturdy frames of type $(2,2)$ algebras provide a new tool in the investigation of semirings.

Because a semiring $(S,+, \cdot)$ is a type $(2,2)$ algebra whose additive and multiplicative reducts are both semigroups and multiplication distributes over addition, we can study the structure of a semiring by using the properties of its reducts. We now call a semiring $(S,+, \cdot)$ an $A$-completely regular semiring if it is a union of some subsemirings whose additive reducts are rectangular groups. We will show that if a type $(2,2)$ algebra $S$ is represented by a sturdy frame of type $(2,2)$ algebras on a frame $B$, then $S$ can be constructed as a subdirect product of the frame $B$ and a type $(2,2)$ factor algebra $S / \theta$, where $\theta$ is a congruence on $S$. This result is particularly useful in the study of varieties of type $(2,2)$ algebras. For example, if $S$ is an A-completely regular semiring, then we can show that $S$ is a subdirect product of a semiring whose additive reduct is a rectangular group and an idempotent semiring with semilattice additive reduct. This is equivalent to saying that $(S,+)$ is a subdirect product of a rectangular group and a semilattice. Some other characterization theorems for idempotent semirings can also be obtained by using sturdy frames of type $(2,2)$ algebras. In fact, many of our results generalize the results recently obtained by S. Ghosh [2].

Throughout this paper, we denote the class of all semirings with completely regular additive reducts by $\mathbf{C R}^{+}$, the class of all semirings with completely simple additive reducts by $\mathbf{C S}^{+}$, the class of all semirings whose additive reducts are rectangular groups by $\mathbf{R e G}{ }^{+}$, the class of all idempotent semirings whose additive reducts are semilattices by $\mathbf{S} \ell^{+}$, and the class of all idempotent semirings whose multiplicative reducts are rectangular bands by $\mathbf{R}^{\bullet}$. We also denote Green's $\mathcal{D}$-relation on the semiring additive [multiplicative $]$ reduct by $\stackrel{+}{\mathcal{D}}[\dot{\mathcal{D}}]$. For other notation and terminology not explained 
in this paper, the reader is referred to Grätzer [3], Howie [5] or Petrich and Reilly [7].

2. Properties of sturdy frames of type $(2,2)$ algebras. In this section, we study some properties of sturdy frames of type $(2,2)$ algebras. We will show that some results on semirings obtained by S. Ghosh [2] are corollaries of our results.

TheOREM 2.1. Let $\mathbf{V}$ be the variety of type $(2,2)$ algebras. If for all $\alpha \in B$, the frame $B$ and the type $(2,2)$ algebras $S_{\alpha}$ are in the variety $\mathbf{V}$, then the sturdy frame $S=\left\langle B, \leq ; S_{\alpha}, \varphi_{\alpha, \beta}\right\rangle$ of the type $(2,2)$ algebras $S_{\alpha}$ on the frame $B$ is also in $\mathbf{V}$.

Proof. We only need to consider a term $T\left(x_{1}, \ldots, x_{n}\right)$ over the set $\left\{x_{1}, \ldots, x_{n}\right\}$. We first claim that for all $a_{i} \in S_{\alpha_{i}}$, we have

$$
T\left(a_{1}, \ldots, a_{n}\right)=T\left(a_{1} \varphi_{\alpha_{1}, \alpha_{0}}, a_{2} \varphi_{\alpha_{2}, \alpha_{0}}, \ldots, a_{n} \varphi_{\alpha_{n}, \alpha_{0}}\right) \varphi_{T\left(\alpha_{1}, \alpha_{2}, \ldots, \alpha_{n}\right), \alpha_{0}}^{-1},
$$

where $\alpha_{0}=\operatorname{lub}\left(\alpha_{1}, \ldots, \alpha_{n}\right) \in B$, and therefore $T\left(a_{1}, \ldots, a_{n}\right) \in S_{T\left(\alpha_{1}, \ldots, \alpha_{n}\right)}$.

In fact, if $T\left(x_{1}, \ldots, x_{n}\right)=x_{i}$, then

$$
\begin{aligned}
T\left(a_{1}, \ldots, a_{n}\right) & =a_{i}=\left(a_{i} \varphi_{\alpha_{i}, \alpha_{0}}\right) \varphi_{\alpha_{i}, \alpha_{0}}^{-1} \\
& =\left(T\left(a_{1} \varphi_{\alpha_{1}, \alpha_{0}}, \ldots, a_{n} \varphi_{\alpha_{n}, \alpha_{0}}\right) \varphi_{T\left(\alpha_{1}, \ldots, \alpha_{n}\right), \alpha_{0}}^{-1} .\right.
\end{aligned}
$$

Hence, our claim is established.

Now, to $T_{1}\left(x_{1}, \ldots, x_{n}\right)$ and $T_{2}\left(x_{1}, \ldots, x_{n}\right)$, we assign the following symbols:

$$
\begin{aligned}
& \alpha=T_{1}\left(\alpha_{1}, \ldots, \alpha_{n}\right), \quad \beta=T_{2}\left(\alpha_{1}, \ldots, \alpha_{n}\right), \\
& T_{1}\left(a_{1}, \ldots, a_{n}\right)=T_{1}\left(a_{1} \varphi_{\alpha_{1}, \alpha_{0}}, \ldots, a_{n} \varphi_{\alpha_{n}, \alpha_{0}}\right) \varphi_{\alpha, \alpha_{0}}^{-1}, \\
& T_{2}\left(a_{1}, \ldots, a_{n}\right)=T_{2}\left(a_{1} \varphi_{\alpha_{1}, \alpha_{0}}, \ldots, a_{n} \varphi_{\alpha_{n}, \alpha_{0}}\right) \varphi_{\beta, \alpha_{0}}^{-1} .
\end{aligned}
$$

Then for $T\left(x_{1}, \ldots x_{n}\right)=T_{1}\left(x_{1}, \ldots, x_{n}\right)+T_{2}\left(x_{1}, \ldots, x_{n}\right)$, we can write

$$
\begin{aligned}
T\left(a_{1}, \ldots, a_{n}\right)= & T_{1}\left(a_{1}, \ldots, a_{n}\right)+T_{2}\left(a_{1}, \ldots, a_{n}\right) \\
= & T_{1}\left(a_{1} \varphi_{\alpha_{1}, \alpha_{0}}, \ldots, a_{n} \varphi_{\alpha_{n}, \alpha_{0}}\right) \varphi_{\alpha, \alpha_{0}}^{-1} \\
& +T_{2}\left(a_{1} \varphi_{\alpha_{1}, \alpha_{0}}, \ldots, a_{n} \varphi_{\alpha_{n}, \alpha_{0}}\right) \varphi_{\beta, \alpha_{0}}^{-1} . \\
=\{ & \left(T_{1}\left(a_{1} \varphi_{\alpha_{1}, \alpha_{0}}, \ldots, a_{n} \varphi_{\alpha_{n}, \alpha_{0}}\right) \varphi_{\alpha, \alpha_{0}}^{-1}\right) \varphi_{\alpha, \alpha \vee \beta} \\
& \left.+\left(T_{2}\left(a_{1} \varphi_{\alpha_{1}, \alpha_{0}}, \ldots, a_{n} \varphi_{\alpha_{n}, \alpha_{0}}\right) \varphi_{\beta, \alpha_{0}}^{-1}\right) \varphi_{\beta, \alpha \vee \beta}\right\} \varphi_{\alpha+\beta, \alpha \vee \beta}^{-1} \\
= & \left\{T_{1}\left(a_{1} \varphi_{\alpha_{1}, \alpha_{0}}, \ldots, a_{n} \varphi_{\alpha_{n}, \alpha_{0}}\right)\left(\varphi_{\alpha, \alpha_{0}}^{-1} \varphi_{\alpha, \alpha \vee \beta}\right)\right. \\
& \left.+T_{2}\left(a_{1} \varphi_{\alpha_{1}, \alpha_{0}}, \ldots, a_{n} \varphi_{\alpha_{n}, \alpha_{0}}\right)\left(\varphi_{\beta, \alpha_{0}}^{-1} \varphi_{\beta, \alpha \vee \beta}\right)\right\} \varphi_{\alpha+\beta, \alpha \vee b \beta}^{-1}
\end{aligned}
$$




$$
\begin{aligned}
=\{ & T_{1}\left(a_{1} \varphi_{\alpha_{1}, \alpha_{0}}, \ldots, a_{n} \varphi_{\alpha_{n}, \alpha_{0}}\right) \varphi_{\alpha_{0}, \alpha \vee \beta} \\
& \left.+T_{2}\left(a_{1} \varphi_{\alpha_{1}, \alpha_{0}}, \ldots, a_{n} \varphi_{\alpha_{n}, \alpha_{0}}\right) \varphi_{\alpha_{0}, \alpha \vee \beta}\right\} \varphi_{\alpha+\beta, \alpha \vee \beta}^{-1} \\
= & \left\{T_{1}\left(a_{1} \varphi_{\alpha_{1}, \alpha_{0}} \varphi_{\alpha_{0}, \alpha \vee \beta}, \ldots, a_{n} \varphi_{\alpha_{n}, \alpha_{0}} \varphi_{\alpha_{0}, \alpha \vee \beta}\right)\right. \\
& \left.+T_{2}\left(a_{1} \varphi_{\alpha_{1}, \alpha_{0}} \varphi_{\alpha_{0}, \alpha,{ }}, \ldots, a_{n} \varphi_{\alpha_{n}, \alpha_{0}} \varphi_{\alpha_{0}, \alpha \vee \beta}\right)\right\} \varphi_{\alpha+\beta, \alpha \vee \beta}^{-1} \\
= & \left\{T_{1}\left(a_{1} \varphi_{\alpha_{1}, \alpha \vee \beta}, \ldots, a_{n} \varphi_{\alpha_{n}, \alpha \vee \beta}\right)\right. \\
& \left.+T_{2}\left(a_{1} \varphi_{\alpha_{1}, \alpha \vee \beta}, \ldots, a_{n} \varphi_{\alpha_{n}, \alpha \vee \beta}\right)\right\} \varphi_{\alpha+\beta, \alpha \vee \beta}^{-1} \\
= & T\left(a_{1} \varphi_{\alpha_{1}, \alpha \vee \beta}, \ldots, a_{n} \varphi_{\alpha_{n}, \alpha \vee \beta}\right) \varphi_{\alpha+\beta, \alpha \vee \beta}^{-1} \\
= & \left(T\left(a_{1} \varphi_{\alpha_{1}, \alpha \vee \beta}, \ldots, a_{n} \varphi_{\alpha_{n}, \alpha \vee \beta}\right)\left(\varphi_{\alpha, \vee \beta, \alpha_{0}} \varphi_{\alpha \vee \beta, \alpha_{0}}^{-1}\right) \varphi_{\alpha+\beta, \alpha \vee \beta}^{-1}\right. \\
= & \left(T\left(a_{1} \varphi_{\alpha_{1}, \alpha \vee \beta}, \ldots, a_{n} \varphi_{\alpha_{n}, \alpha \vee \beta}\right) \varphi_{\alpha \vee \beta, \alpha_{0}}\right)\left(\varphi_{\alpha \vee \beta, \alpha_{0}}^{-1} \varphi_{\alpha+\beta, \alpha \vee \beta}^{-1}\right) \\
= & T\left(a_{1} \varphi_{\alpha_{1}, \alpha \vee \beta} \varphi_{\alpha \vee \beta, \alpha_{0}}, \ldots, a_{n} \varphi_{\alpha_{n}, \alpha \vee \beta} \varphi_{\alpha \vee \beta, \alpha_{0}}\right)\left(\varphi_{\alpha+\beta, \alpha \vee \beta} \varphi_{\alpha \vee \beta, \alpha_{0}}\right)^{-1} \\
= & T\left(a_{1} \varphi_{\alpha_{1}, \alpha_{0}}, \ldots, a_{n} \varphi_{\alpha_{n}, \alpha_{0}}\right) \varphi_{\alpha+\beta, \alpha_{0}}^{-1} \\
= & T\left(a_{1} \varphi_{\alpha_{1}, \alpha_{0}}, \ldots, a_{n} \varphi_{\alpha_{n}, \alpha_{0}}\right) \varphi_{T\left(\alpha_{1}, \alpha_{2}, \ldots, \alpha_{n}\right), \alpha_{0}}^{-1} .
\end{aligned}
$$

For $T=T_{1} \cdot T_{2}$, we can similarly write

$$
T\left(a_{1}, \ldots, a_{n}\right)=T\left(a_{1} \varphi_{\alpha_{1}, \alpha_{0}}, \ldots, a_{n} \varphi_{\alpha_{n}, \alpha_{0}}\right) \varphi_{T\left(\alpha_{1}, \ldots, \alpha_{n}\right), \alpha_{0}}^{-1} .
$$

As a consequence of our claim, we can easily see that if the frame $(B,+, \cdot)$ and each type $(2,2)$ algebra $\left(S_{\alpha},+, \cdot\right)(\alpha \in B)$ satisfy an identity then the sturdy frame $S$ also satisfies the same identity. Thus, Theorem 2.1 is proved.

By Theorem 2.1, we can immediately see that if the frame $B$ and all type $(2,2)$ algebras $S_{\alpha}(\alpha \in B)$ are semirings, then the sturdy frame $\left\langle B, \leq ; S_{\alpha}, \varphi_{\alpha, \beta}\right\rangle$ is also a semiring. In particular, Theorem 1.2 of [2] becomes a special case of our Theorem 2.1 when the frame $B$ is a distributive lattice on which the upper semilattice order $\leq$ is defined by

$$
a \leq b \text { if and only if } a+b=b,
$$

and all the semirings $S_{\alpha}$ are rings.

Theorem 2.2. Define a binary relation $\theta$ on $S=\left\langle B, \leq ; S_{\alpha}, \varphi_{\alpha, \beta}\right\rangle$ by

$$
a \theta b \Leftrightarrow a \varphi_{\alpha, \alpha \vee \beta}=b \varphi_{\beta, \alpha \vee \beta}
$$

for any $a, b \in S$. Then $\theta$ is a congruence on $S$ and $S$ is a subdirect product of $B$ and $S / \theta$.

Proof. It is clear that $\theta$ is reflexive and symmetric. To see that it is transitive, we let $a \in S_{\alpha}, b \in S_{\beta}$, and $c \in S_{\gamma}$, where $\alpha, \beta, \gamma \in B$. Assume 
that $a \theta b$ and $b \theta c$. Then

$$
a \varphi_{\alpha, \alpha \vee \beta}=b \varphi_{\beta, \alpha \vee \beta}, \quad b \varphi_{\beta, \beta \vee \gamma}=c \varphi_{\gamma, \beta \vee \gamma}
$$

Consequently,

$$
\begin{aligned}
a \varphi_{\alpha, \alpha \vee \beta \vee \gamma} & =a \varphi_{\alpha, \alpha \vee \beta} \varphi_{\alpha \vee \beta, \alpha \vee \beta \vee \gamma}=b \varphi_{\beta, \alpha \vee \beta} \varphi_{\alpha \vee \beta, \alpha \vee \beta \vee \gamma} \\
& =b \varphi_{\beta, \alpha \vee \beta \vee \gamma}=b \varphi_{\beta, \beta \vee \gamma} \varphi_{\beta \vee \gamma, \alpha \vee \beta \vee \gamma} \\
& =c \varphi_{\gamma, \beta \vee \gamma} \varphi_{\beta \vee \gamma, \alpha \vee \beta \vee \gamma}=c \varphi_{\gamma, \alpha \vee \beta \vee \gamma}
\end{aligned}
$$

Thus,

$$
\begin{aligned}
a \varphi_{\alpha, \alpha \vee \gamma} & =a\left(\varphi_{\alpha, \alpha \vee \beta \vee \gamma} \varphi_{\alpha \vee \gamma, \alpha \vee \beta \vee \gamma}^{-1}\right)=\left(a \varphi_{\alpha, \alpha \vee \beta \vee \gamma}\right) \varphi_{\alpha \vee \gamma, \alpha \vee \beta \vee \gamma}^{-1} \\
& =\left(c \varphi_{\gamma, \alpha \vee \beta \vee \gamma}\right) \varphi_{\alpha \vee \gamma, \alpha \vee \beta \vee \gamma}^{-1}=c \varphi_{\gamma, \alpha \vee \gamma}
\end{aligned}
$$

This shows that $a \theta c$ and hence $\theta$ is transitive. In other words, $\theta$ is an equivalence relation on $S$.

To show that $\theta$ is a congruence relation on $S$, we first assume that $a \theta b$. Then $a \varphi_{\alpha, \alpha \vee \beta}=b \varphi_{\beta, \alpha \vee \beta}$. Now, for the term $T\left(x_{1}, x_{2}\right)=x_{1}+x_{2}, x_{2}+x_{1}$, $x_{1} x_{2}$, or $x_{2} x_{1}$, we have

$$
\begin{aligned}
& T(a, c) \varphi_{T(\alpha, \gamma)}, \alpha \vee \beta \vee \gamma \\
&=\left(T\left(a \varphi_{\alpha, \alpha \vee \gamma}, c \varphi_{\gamma, \alpha \vee \gamma}\right) \varphi_{T(\alpha, \gamma), \alpha \vee \gamma}^{-1}\right) \varphi_{T(\alpha, \gamma), \alpha \vee \beta \vee \gamma} \\
&=\left(T\left(a \varphi_{\alpha, \alpha \vee \gamma}, c \varphi_{\gamma, \alpha \vee \gamma}\right) \varphi_{T(\alpha, \gamma), \alpha \vee \gamma}^{-1}\right) \varphi_{T(\alpha, \gamma), \alpha \vee \gamma} \varphi_{\alpha \vee \gamma, \alpha \vee \beta \vee \gamma} \\
&=T\left(a \varphi_{\alpha, \alpha \vee \gamma}, c \varphi_{\gamma, \alpha \vee \gamma}\right) \varphi_{\alpha \vee \gamma, \alpha \vee \beta \vee \gamma} \\
&=T\left(a \varphi_{\alpha, \alpha \vee \gamma} \varphi_{\alpha \vee \gamma, \alpha \vee \beta \vee \gamma}, c \varphi_{\gamma, \alpha \vee \gamma} \varphi_{\alpha \vee \gamma, \alpha \vee \beta \vee \gamma}\right) \\
&=T\left(a \varphi_{\alpha, \alpha \vee \beta \vee \gamma}, c \varphi_{\gamma, \alpha \vee \beta \vee \gamma}\right) \\
&=T\left(a \varphi_{\alpha, \alpha \vee \beta} \varphi_{\alpha \vee \beta, \alpha \vee \beta \vee \gamma}, c \varphi_{\gamma, \alpha \vee \beta \vee \gamma}\right) \\
&=T\left(b \varphi_{\beta, \alpha \vee \beta} \varphi_{\alpha \vee \beta, \alpha \vee \beta \vee \gamma}, c \varphi_{\gamma, \alpha \vee \beta \vee \gamma}\right) \\
&=T\left(b \varphi_{\beta, \alpha \vee \beta \vee \gamma}, c \varphi_{\gamma, \alpha \vee \beta \vee \gamma}\right)=T(b, c) \varphi_{T(\beta, \gamma), \alpha \vee \beta \vee \gamma}
\end{aligned}
$$

This leads to

$$
\begin{aligned}
T(a, c) \varphi_{T(\alpha, \gamma), T(\alpha, \gamma) \vee T(\beta \gamma)} & =T(a, c) \varphi_{T(\alpha, \gamma), \alpha \vee \beta \vee \gamma} \varphi_{T(\alpha, \gamma) \vee T(\beta \gamma), \alpha \vee \beta \vee \gamma}^{-1} \\
& =T(b, c) \varphi_{T(\beta, \gamma), \alpha \vee \beta \vee \gamma} \varphi_{T(\alpha, \gamma) \vee T(\beta \gamma), \alpha \vee \beta \vee \gamma}^{-1} \\
& =T(b, c) \varphi_{T(\beta, \gamma), T(\alpha, \gamma) \vee T(\beta \gamma)} .
\end{aligned}
$$

Thus, we have shown that $T(a, c) \theta T(b, c)$, and $\theta$ is a congruence on $S=$ $\bigcup_{\alpha \in B} S_{\alpha}$.

Finally, we can easily verify that the mapping $\psi: S \rightarrow B \times(S / \theta)$, defined by $a \psi=(\alpha, a \theta)$ for any $a \in S_{\alpha}(\alpha \in B)$, is a monomorphism, and also the projections which map $S \psi$ onto $B$ and $S / \theta$ respectively are homomorphisms. Therefore, $S$ is a subdirect product of $B$ and $S / \theta$. 
REMARK. In Theorem 2.2, we can see that $S / \theta$ is a direct limit of certain subalgebras $\mathcal{A}=\left\langle A, \leq ; S_{\alpha}, \varphi_{\alpha, \beta}\right\rangle$ [4, pp. 128-139].

Since $B$ is a factor of the subdirect product decomposition of $S$ by Theorem 2.2 and every $S_{\alpha}$ is a subalgebra of $S$, we see that if $S$ satisfies a given identity, then $B$ and every $S_{\alpha}$ also satisfy the same identity. Thus, using Theorem 2.1, we deduce the following theorem.

Theorem 2.3. The frame $(B,+, \cdot)$ and every type $(2,2)$ algebra $\left(S_{\alpha},+, \cdot\right)$ $(\alpha \in B)$ satisfy a given identity if and only if the sturdy frame $\left\langle B, \leq ; S_{\alpha}, \varphi_{\alpha, \beta}\right\rangle$ also satisfies the same identity.

3. A-completely regular semirings. Recall that a completely regular semigroup is a union of groups. In particular, a semigroup is completely regular if and only if it is a semilattice of completely simple semigroups. An orthodox completely simple semigroup is called a rectangular group. We will use the symbols $\mathbf{C R}^{+}, \mathbf{C S}^{+}$and $\mathbf{S} \ell^{+}$to denote the classes of semirings defined in the introduction.

We define $\mathbf{S}=\mathbf{W} \circ \mathbf{V}$ to be the Mal'tsev product of the classes $\mathbf{W}$ and $\mathbf{V}$ of semirings if there exists a congruence $\varrho$ on $S$ with $S / \varrho \in \mathbf{V}$ and all $\varrho$-classes that are subsemirings are in $\mathbf{W}$.

We now study the Mal'tsev product of two important classes of semirings, namely $\mathbf{C S}^{+}$and $\mathbf{S} \ell^{+}$.

Lemma 3.1. The following conditions on a semiring $S$ are equivalent.

(i) $S$ is a union of subsemirings which are members of $\mathbf{C S}^{+}$.

(ii) $S$ is a disjoint union of subsemirings which are the members of $\mathbf{C S}^{+}$.

(iii) $S$ is in the Mal'tsev product $\mathbf{C S}^{+} \circ \mathbf{S} \ell^{+}$.

(iv) $S$ is in $\mathbf{C R}^{+}$and every $\stackrel{+}{\mathcal{D}}$-class of $S$ is a subsemiring of $S$.

Proof. (iii) $\Rightarrow$ (ii) and (ii) $\Rightarrow$ (i) are trivial. To prove that (iv) $\Rightarrow$ (iii), suppose that (iv) holds. Then the semiring $S$ is in $\mathbf{C R}^{+}$, i.e., $(S,+)$ is a completely regular semigroup. In this case, we immediately see that Green's $\stackrel{+}{\mathcal{D}}$ relation is a congruence on $S$. Also, by our assumption, every $\stackrel{+}{\mathcal{D}}$-class of $S$ is a subsemiring of $S$. Hence every $\stackrel{+}{\mathcal{D}}$-class of $S$ is in $\mathbf{C S}^{+}$and so the factor semiring $S / \stackrel{\mathcal{D}}{ }^{+}$is an idempotent semiring in $\mathbf{S} \ell^{+}$. This implies that $S$ is in $\mathbf{C S}^{+} \circ \mathbf{S} \ell^{+}$.

(i) $\Rightarrow$ (iv). Assume that $S$ is the union of subsemirings $S_{\alpha}$ which are members of $\mathbf{C S}^{+}$. Then $S$ is in $\mathbf{C R}^{+}$. Also, since each $S_{\alpha}$ is in $\mathbf{C S}^{+}, S_{\alpha}$ is contained in a $\stackrel{+}{\mathcal{D}}$-class of $S$. This implies that every $\stackrel{+}{\mathcal{D}}$-class of $S$ is a subsemiring of $S$. 
Definition 3.2. A semiring is said to be A-completely regular if it satisfies the conditions in Lemma 3.1.

Semirings which are unions of rings are special A-completely regular semirings. The structure of A-completely regular semirings has recently been investigated by Pastijn and Guo [6].

LEMma 3.3. If we define an upper semilattice order $\leq$ on a semiring $B \in \mathbf{S} \ell^{+}$by

$$
a \leq b \Leftrightarrow a+b=b
$$

then $B$ becomes a frame.

Proof. Assume that $B \in \mathbf{S} \ell^{+}$, i.e, $B$ is an idempotent semiring whose additive reduct is indeed a semilattice. Define an upper semilattice order on $B$ as in the lemma. Then

$$
(a+b)+a \vee b=a+(b+a \vee b)=a+a \vee b=a \vee b
$$

and

$$
\begin{aligned}
a b+a \vee b & =a b+(a+b+a \vee b)=(a b+a+b)+a \vee b \\
& =\left(a b+(a+b)^{2}\right)+a \vee b=\left(a b+a^{2}+a b+b a+b^{2}\right)+a \vee b \\
& =(a+a b+b a+b)+a \vee b=(a+b)+a \vee b=a \vee b .
\end{aligned}
$$

These equalities imply that $a+b \leq a \vee b$ and $a b \leq a \vee b$. Hence, $B$ is a frame, as required.

Lemma 3.4. Let $S$ be an A-completely regular semiring. Then $S$ is a sturdy frame $\left\langle B, \leq ; S_{\alpha}, \varphi_{\alpha, \beta}\right\rangle$ of semirings in the class $\mathbf{R e G} \mathbf{G}^{+}$, where $B$ is the frame as described in Lemma 3.3, if and only if the additive reduct $(S,+)$ is a sturdy semilattice of rectangular groups.

Proof. The necessity part is evident. We only need to prove the sufficiency. Let $S$ be an A-completely regular semiring. Assume that $(S,+)$ is a sturdy semilattice of rectangular groups. Then, by Theorem IV.1.6, Lemma IV.1.7, and Theorem IV.3.6 of [1], we can express $(S,+)=$ $\left\langle B ; S_{\alpha}, \varphi_{\alpha, \beta}\right\rangle$ as a sturdy semilattice of additive semigroups $S_{\alpha}$, where $B$ is the quotient semiring $S / \stackrel{+}{\mathcal{D}}$ and each $S_{\alpha}$ is a $\stackrel{+}{\mathcal{D}}$-class of $S$; moreover, each $\varphi_{\alpha, \beta}$ is a monomorphism which maps the rectangular group $\left(S_{\alpha},+\right)$ into $\left(S_{\beta},+\right)$, and $a \varphi_{\alpha, \beta}$ is the unique element of $S_{\beta}$ satisfying $a \leq a \varphi_{\alpha, \beta}$.

Now, by Lemma 3.1, we immediately see that $B=S / \stackrel{+}{\mathcal{D}}$ is an idempotent semiring. Furthermore, according to Lemma $3.3, B$ is a frame.

On the other hand, by our assumption and Lemma 3.1, we can see that the $\stackrel{+}{\mathcal{D}}$-classes of $S$, i.e., the semirings $S_{\alpha}$, are members of $\mathbf{C S}^{+}$.

We now prove that every $\varphi_{\alpha, \beta}$ is a monomorphism from $S_{\alpha}$ to $S_{\beta}$. For any $\alpha, \beta \in B$ with $\alpha \leq \beta$ and $a, b \in S_{\alpha}, c, d \in S_{\beta}$ satisfying $a \leq c$ (i.e., 
$a \varphi_{\alpha, \beta}=c$ ) and $b \leq d$ (i.e., $b \varphi_{\alpha, \beta}=d$ ), we may take $e, f, g, h \in E^{+}\left(S_{\beta}\right)$ such that $c=e+a=a+g$ and $d=f+b=b+h$. Thus, we have $c d=(e+a)(f+b)=e f+a f+e b+a b=(a+g)(b+h)=a b+g b+a h+g h$. By Lemma 1.1 in [6], we see that every element in the set $\{e f, a f, e b, g b, a h, g h\}$ is in $E^{+}(S)$. On the other hand, by Theorem II.5.3 and Corollary III.5.3 of [1], we see that $E^{+}(S)$ forms a subband of $(S,+)$. Hence, both $e f+a f+e b$ and $g b+a h+g h$ are in $E^{+}(S)$. Furthermore, we get $c d \leq a b$. This implies that

$$
a \varphi_{\alpha, \beta} b \varphi_{\alpha, \beta}=c d=a b \varphi_{\alpha, \beta}
$$

and hence every mapping $\varphi_{\alpha, \beta}$ is a semiring monomorphism from $S_{\alpha}$ to $S_{\beta}$.

Finally, it is trivial to see that for any $a \in S_{\alpha}, b \in S_{\beta}$, we have

$$
a+b=\left(a \varphi_{\alpha, \alpha \vee \beta}+b \varphi_{\beta, \alpha \vee \beta}\right) \varphi_{\alpha+\beta, \alpha \vee \beta}^{-1}, \quad a b=\left(a \varphi_{\alpha, \alpha \vee \beta} b \varphi_{\beta, \alpha \vee \beta}\right) \varphi_{\alpha \beta, \alpha \vee \beta}^{-1} .
$$

This proves that $S=\left\langle B, \leq ; S_{\alpha}, \varphi_{\alpha, \beta}\right\rangle$ is indeed a sturdy frame of semirings $S_{\alpha}$, where every $S_{\alpha}$ is in $\mathbf{R e G}^{+}$.

As a consequence of Lemma 3.4, we can deduce the following characterization theorem for $\mathbf{R e G}{ }^{+}$semirings.

TheOREm 3.5. Let $S$ be an A-completely regular semiring. Then $S$ is a subdirect product of a semiring in the class $\mathbf{R e G} \mathbf{G}^{+}$and another semiring in the class $\mathbf{S} \ell^{+}$if and only if the additive reduct $(S,+)$ is a subdirect product of a rectangular group and a semilattice.

Proof. The necessity part is evident. We only need to prove the sufficiency. Assume that $S$ is an A-completely regular semiring, and $(S,+)$ is a subdirect product of a rectangular group and a semilattice. Then, by Theorem II.5.3, Corollary III.5.3 and Theorem IV.3.6 of [1], $(S,+)$ is a sturdy semilattice of rectangular groups. Then, by Lemma $3.4, S=\left\langle B, \leq ; S_{\alpha}, \varphi_{\alpha, \beta}\right\rangle$ is a sturdy frame of semirings, where $B$ is the frame described in Lemma 3.3 and each semiring $S_{\alpha}$ is a $\stackrel{+}{\mathcal{D}}$-class of $S$ in the class $\mathbf{R e G}{ }^{+}$. Also, if we define a binary relation $\theta$ on $S$ by

$$
a \theta b \Leftrightarrow a \varphi_{\alpha, \alpha \vee \beta}=b \varphi_{\beta, \alpha \vee \beta}
$$

then, by Theorem 2.2, $\theta$ is a congruence on $S$. Consequently, $S$ can be expressed as a subdirect product of $B$ and $S / \theta$. Also, it can be easily seen that $(S / \theta,+)$ is a rectangular group, as required.

By using the concept of the sturdy frame of type $(2,2)$ algebras, we are now able to give another proof of Lemma 3.4 of [6].

We first let $A$ be a semiring in the Mal'tsev product $\mathbf{R i} \circ \mathbf{S} \ell^{+}$, where $\mathbf{R i}$ denotes the class of all rings. Then Green's $\mathcal{H}^{+}$-relation on the additive reduct $(A,+)$ is the least $\mathbf{S} \ell^{+}$-congruence on $A$, and $I=A / \mathcal{H}^{+} \in \mathbf{S} \ell^{+}$is the frame described in Lemma 3.3. Now, we consider the ring $R_{A}$ associated 
with the semiring $A$, defined by Pastijn and Guo [6]. For any $\alpha \in I$, we let

$$
S_{\alpha}^{\prime}=\left\{\sum_{i \in I} a_{i} \in R_{A} \mid \text { if } a_{i} \neq 0_{i}, \text { then } i \leq \alpha\right\}
$$

and $S_{\alpha}=\left(\alpha, S_{\alpha}^{\prime}\right)$. We can see that every $S_{\alpha}^{\prime}$ is clearly a subring of $R_{A}$ and $S_{\alpha} \subseteq S_{\beta}$ for any $\alpha \leq \beta \in I$. Clearly, every $S_{\alpha}^{\prime}$ with coordinatewise operations is a ring which is isomorphic to $S_{\alpha}$. We now define a monomorphism $\varphi_{\alpha, \beta}$ of $S_{\alpha}^{\prime}$ to $S_{\beta}^{\prime}$ for $\alpha \leq \beta$ by $\varphi_{\alpha, \beta}\left(\alpha, \sum_{i \in I} a_{i}\right)=\left(\beta, \sum_{i \in I} a_{i}\right)$. Then, by Theorem 2.2, the sturdy frame $\left\langle I, \leq ; S_{\alpha}, \varphi_{\alpha, \beta}\right\rangle$ is a subdirect product of $I$ and $R_{A}$. Moreover, we can easily check that it is exactly the semiring $T_{A}$ of [6]. This shows that Lemma 3.4 of [6] holds.

As an analogy of a well known result that every Clifford semigroup has an E-unitary cover (Lemma IV.4.4 of [7]), Pastijn and Guo [6] also defined a homomorphism $\varphi$ of the semiring $T_{A}$ to the semiring $A$ so that $\varphi$ separates the additive idempotents of the semiring $A$ (Lemma 3.5 of [6]). The above results are particularly important in the study of the semiring variety $\mathbf{O}$ introduced by Pastijn and Guo [6].

4. Semirings whose additive reducts are semilattices. In this section, we study the structure of idempotent semirings whose additive reducts are semilattices. A structure theorem for this kind of idempotent semirings is given.

LEMMA 4.1. Let $S$ be an idempotent semiring whose additive reduct is a semilattice, i.e., $S \in \mathbf{S} \ell^{+}$. Then $S$ satisfies the identities

$$
x+y+x y=x+y, \quad x+y+y x=x+y .
$$

Proof. The proof is straightforward and is hence omitted.

A (meet) distributive bisemilattice $B$ is an idempotent semiring satisfying the following identities:

$$
x+y=y+x, \quad x y=y x .
$$

As an analogy of Lemma 3.3, we have the following lemma.

Lemma 4.2. Let $B$ be a distributive bisemilattice. If $\leq i$ is an upper semilattice order on $B$ defined by

$$
a \leq b \Leftrightarrow a b=b,
$$

then $B$ is a frame.

THEOREM 4.3. Let $S$ be an idempotent semiring whose additive reduct is a semilattice. Then $S$ can be expressed as a sturdy frame $\left\langle B, \leq ; S_{\alpha}, \varphi_{\alpha, \beta}\right\rangle$ of semirings $S_{\alpha}$ in the class $\mathbf{R}^{\bullet}$ on the frame $B$ as described in Lemma 4.2 if and only if $(S, \cdot)$ is a sturdy semilattice of rectangular bands. 
Proof. The necessity part is evident. For the sufficiency, we consider an idempotent semiring $S$ whose mutiplicative reduct $(S, \cdot)$ is a sturdy semilattice of rectangular bands. Also, we may assume that the semigroup $(S, \cdot)=\left\langle B ; S_{\alpha}, \varphi_{\alpha, \beta}\right\rangle$ is a sturdy semilattice $B$ of rectangular bands $S_{\alpha}$, where $B=S / \dot{\mathcal{D}}$ and each $S_{\alpha}$ is a $\dot{\mathcal{D}}$-class of $S$ and each $\varphi_{\alpha, \beta}(\alpha \leq \beta)$, defined by $a \varphi_{\alpha, \beta}=b$ for the unique element $b$ of $S_{\beta}$ such that $a b=b a=b$, is a monomorphism from the rectangular band $\left(S_{\alpha}, \cdot\right)$ to the rectangular band $\left(S_{\beta}, \cdot\right)$ (refer to Proposition IV.6.1 of [1], and Propositions 4.6.13 and 4.6.14 of $[2])$.

To prove that $S=\left\langle B ; S_{\alpha}, \varphi_{\alpha, \beta}\right\rangle$ is a sturdy frame of some members $S_{\alpha}$ of $\mathbf{R}^{\bullet}$ on the frame $B$ as described in Lemma 4.2, we need to prove that $\dot{\mathcal{D}}$ is also a congruence on the semiring $S$. Since $(S, \cdot)$ is a normal band, it satisfies the identity $x y z x=x z y x$. Thus, if $a \dot{\mathcal{D}} b$, by Lemma 4.1 , then

$$
\begin{aligned}
(a+c)(b+c)(a+c) & =(a b+c b+a c+c)(a+c) \\
& =a b a+c b a+a c a+c a+a b c+c b c+a c+c \\
& =(a+a c a+c a+a c+c)+c b a+a b c+c b c \\
& =a+c+(a c+a c a+c a)+c b a+a b c+c b c \\
& =a+c+c b a+a b c+c b c \\
& =a+c+c a b a+a b a c+c b a b c \\
& =a+c+c a+a c+c a b c \\
& =a+c+c a b a c=a+c+c a c=a+c
\end{aligned}
$$

for any $a, b, c \in S$. Also, by interchanging the roles of $a$ and $b$, we obtain $(b+c)(a+c)(b+c)=b+c$, and consequently, $a+c \dot{\mathcal{D}} b+c$. This shows that $\dot{\mathcal{D}}$ is a congruence on the semiring $S$. Moreover, it can be easily seen that $B=S / \dot{\mathcal{D}}$ is a semiring so that $B$ turns out to be a frame as described in Lemma 4.2. On the other hand, each $\dot{\mathcal{D}}$-class of $S$ is an idempotent semiring in $\mathbf{R}^{\bullet}$.

Since $(S, \cdot)$ is a sturdy semilattice of rectangular bands, we may assume that the semigroup $(S, \cdot)=\left\langle B ; S_{\alpha}, \varphi_{\alpha, \beta}\right\rangle$ is a sturdy semilattice $B$ of rectangular bands $S_{\alpha}$, where $B=S / \mathcal{D}$ and each $S_{\alpha}$ is a $\mathcal{D}$-class of $S$ and each $\varphi_{\alpha, \beta}(\alpha \leq \beta)$ is defined by $a \varphi_{\alpha, \beta}=b$ for the unique element $b$ of $S_{\beta}$ such that $a b=b a=b$. Also, it follows from Proposition 4.6.14 of [5] and Proposition IV.6.1 of [7] that every mapping $\varphi_{\alpha, \beta}(\alpha \leq \beta \in B)$ is a semigroup monomorphism from the rectangular band $\left(S_{\alpha}, \cdot\right)$ into the rectangular band $\left(S_{\beta}, \cdot\right)$ such that for any $\alpha, \beta, \gamma \in B$, we have

(i) $\varphi_{\alpha, \alpha}=1_{S_{\alpha}}$;

(ii) if $\alpha \leq \beta \leq \gamma$, then $\varphi_{\alpha, \beta} \varphi_{\beta, \gamma}=\varphi_{\alpha, \gamma}$.

We will next show that for any $a \in S_{\alpha}$ and $b \in S_{\beta}$ with $\alpha, \beta \leq \gamma \in B$, 
we have

$$
a \varphi_{\alpha, \gamma}+b \varphi_{\beta, \gamma}=(a+b) \varphi_{\alpha+\beta, \gamma} .
$$

In fact, by the definition of $\varphi$ and Lemma 4.1, we can derive the following:

$$
\begin{aligned}
(a & +b)\left(a \varphi_{\alpha, \gamma}+b \varphi_{\beta, \gamma}\right) \\
& =a\left(a \varphi_{\alpha, \gamma}\right)+a\left(b \varphi_{\beta, \gamma}\right)+b\left(a \varphi_{\alpha, \gamma}\right)+b\left(b \varphi_{\beta, \gamma}\right) \\
& =\left(a \varphi_{\alpha, \gamma}\right)+a\left(b \varphi_{\beta, \gamma}\right)+b\left(a \varphi_{\alpha, \gamma}\right)+\left(b \varphi_{\beta, \gamma}\right) \\
& =a \varphi_{\alpha, \gamma}+a\left(b \varphi_{\beta, \gamma}\right)\left(a \varphi_{\alpha, \gamma}\right)\left(b \varphi_{\beta, \gamma}\right)+b\left(a \varphi_{\alpha, \gamma}\right)\left(b \varphi_{\beta, \gamma}\right)\left(a \varphi_{\alpha, \gamma}\right)+b \varphi_{\beta, \gamma} \\
& =a \varphi_{\alpha, \gamma}+a\left(a \varphi_{\alpha, \gamma}\right)\left(b \varphi_{\beta, \gamma}\right)\left(b \varphi_{\beta, \gamma}\right)+b\left(b \varphi_{\beta, \gamma}\right)\left(a \varphi_{\alpha, \gamma}\right)\left(a \varphi_{\alpha, \gamma}\right)+b \varphi_{\beta, \gamma} \\
& =a \varphi_{\alpha, \gamma}+\left(a \varphi_{\alpha, \gamma}\right)\left(b \varphi_{\beta, \gamma}\right)+\left(b \varphi_{\beta, \gamma}\right)\left(a \varphi_{\alpha, \gamma}\right)+b \varphi_{\beta, \gamma} \\
& =a \varphi_{\alpha, \gamma}+b \varphi_{\beta, \gamma} .
\end{aligned}
$$

Dually, we also have

$$
\left(a \varphi_{\alpha, \gamma}+b \varphi_{\beta, \gamma}\right)(a+b)=a \varphi_{\alpha, \gamma}+b \varphi_{\beta, \gamma} .
$$

Therefore,

$$
a \varphi_{\alpha, \gamma}+b \varphi_{\beta, \gamma}=(a+b) \varphi_{\alpha+\beta, \gamma} .
$$

Also, if we put $\alpha=\beta$ in the above formula, then it follows immediately that every $\varphi_{\alpha, \beta}$ is indeed a monomorphism from the semiring $S_{\alpha}$ to the semiring $S_{\beta}$. This shows that conditions (1) and (3) in the definition of a sturdy frame of type $(2,2)$ algebras hold.

On the other hand, we can easily see that for any $a \in S_{\alpha}, b \in S_{\beta}$ and $\alpha, \beta \leq \gamma \in B$, we have

$$
a \varphi_{\alpha, \gamma} b \varphi_{\beta, \gamma}=(a b) \varphi_{\alpha \beta, \gamma}
$$

and so conditions (2) and (4) are also satisfied.

As a consequence of Theorem 4.3, we obtain immediately the following corollary, which extends the results of Ghosh [2] and Bandelt and Petrich [1].

COROLlaRY 4.4. Let $S$ be an idempotent semiring whose additive reduct is a semilattice. Then $S$ is a subdirect product of a bisemilattice and an idempotent semiring in $\mathbf{R}^{\bullet}$ if and only if $(S, \cdot)$ is a subdirect product of a semilattice and a rectangular band.

Acknowledgments. The authors would like to thank the referee for his valuable comments on this paper.

\section{References}

[1] H. J. Bandelt and M. Petrich, Subdirect products of rings and distributive lattices, Proc. Edinburgh Math. Soc. 25 (1982), 155-171. 
[2] S. Ghosh, A characterization of semirings which are subdirect products of a distributive lattice and a ring, Semigroup Forum 59 (1999), 106-120.

[3] G. Grätzer, Universal Algebra, Springer, New York, 1979.

[4] Y. Q. Guo, K. P. Shum, and M. K. Sen, Semigroup structure of left C-semirings, Acta Math. Sinica (2003), to appear.

[5] J. M. Howie, Fundamentals of Semigroup Theory, Clarendon Press, Oxford, 1995.

[6] F. Pastijn and Y. Q. Guo, Semirings which are union of rings, Sci. in China (Ser. A) 45 (2002), 172-195.

[7] M. Petrich and N. R. Reilly, Completely Regular Semigroups, Wiley, New York, 1999.

[8] A. Romanowska, Free idempotent distributive semirings with a semilattice reduct, Math. Japon. 27 (1982), 483-493.

[9] M. K. Sen, Y. Q. Guo, and K. P. Shum, A class of idempotent semirings, Semigroup Forum 60 (2000), 351-367.

Department of Mathematics

Northwest University

Xi'an, Shanxi, 710069, P.R. China

E-mail: xianzhongzhao@263.net

Science Faculty

The Chinese University of Hong Kong

Shatin, Hong Kong

E-mail: kpshum@math.cuhk.edu.hk
Institute of Mathematics Southwest China Normal University Beibei, Chongqing 400715, P.R. China E-mail: yqguo@swnu.edu.cn

Received 9 January 2003;

in revised form 29 September 2003 